\title{
A Proposed Method for Producing Embossed Dots Graphics with a 3D Printer
}

\author{
Kazunori Minatani $\left.{ }^{(}\right)$ \\ National Center for University Entrance Examinations, Komaba 2-19-23, \\ Meguro-ku, Tokyo 153-8501, Japan \\ minatani@rd.dnc.ac.jp
}

\begin{abstract}
This study examines embossed dots graphics produced with a $3 \mathrm{D}$ printer for the purpose of providing practical information to visually impaired people. Using 3D printing to produce tactile graphics has a benefit to make use of our cumulative assets in tactile graphics production: techniques and production tools. The author designed this study to specifically take up the following challenge: develop a system that would convert data created using embossed dots graphics drawing software for a braille embosser into data that could be used with 3D CAD software. The conversion would allow for completely customization of the size and shape of every dot, freeing us from the limitations in dot type associated with the braille embosser. Results are confirmed via physical measurements and tactile observation assessments.
\end{abstract}

Keywords: Visually impaired people $\cdot 3 \mathrm{D}$ printer $\cdot$ Tactile graphics Embossed dots graphics

\section{State of the Art and Research Aims}

This study examines embossed dots graphics produced with a 3D printer for the purpose of providing practical information and educational materials to visually impaired people. The interest in providing visually impaired people with tactile observation materials has led to more active interest in attempting to make use of digital fabrication technologies. Along with these developments have been attempts to produce models of things like astronomical bodies or minute plankton [1], which cannot be tactilely observed directly. Researchers have also been working to produce topographical maps in sharp relief. [2] Unfortunately, the 3D solid model products of such researches and development have yet to be widely used in real-world situations. For the most part, graphical information is provided to visually impaired people in these practical settings via the raised paper methods collectively referred to as tactile graphics.

The significant benefit of using $3 \mathrm{D}$ printing to produce tactile graphics is the ability to make use of our cumulative assets in tactile graphics production. 
These assets can be roughly classified as either know-how in expressive technique which is regularized [4] or production tools. In terms of production tools, we can create tactile graphics with a $3 \mathrm{D}$ printer more efficiently if we use production tools specifically designed to create tactile graphics-particularly drawing software. There are three key benefits to tactile graphics produced with computer software: firstly it is easy to edit the designs, secondly the results can theoretically be replicated endlessly, and thirdly the data can be modified and reused. We can enjoy these benefits of using computer software even if we make tactile graphics with a $3 \mathrm{D}$ printer.

Now I'll take a closer look at the advantages that tactile graphics made with a 3D printer have over conventional tactile graphics, particularly over embossed dots graphics made using a braille embosser. I'll use the term " $3 \mathrm{D}$ embossed dots graphics" to refer to the embossed dots tactile graphics that we produced with a 3D printer. These are the main focus of this study. Conventional embossed dots graphics made using a braille embosser will be referred to as "paper embossed dots graphics". The biggest advantage that 3D embossed dots graphics have over paper embossed dots graphics is the ability to freely customize their arrangement namely dots' shapes. Braille embossers primarily use a hammer solenoid to create raised dots on paper, punching out braille as text. The same mechanical process is used when producing embossed dots graphics. Creating embossed dots graphics with a $3 \mathrm{D}$ printer does not have the same limitations as using a braille embosser when it comes to the types of dots that can be created.

There is a research to apply modeling with a $3 \mathrm{D}$ printer to production of thermoform tactile materials. [3] This study is similarly interested in the use of 3D printing in comparison with tactile graphics, but takes the idea a step further by introducing three-dimensional molding as a method of producing and enhancing the expressive power of the embossed dots graphics, an established form of tactile graphics. To summarize, the benefits of this project is that they make it possible to achieve the expressive power of thermoform plus the ease of design that comes with computer-powered embossed dots graphics. My practical aim with this study is to create a system that makes it possible to take the expertise developed among embossed dots graphics designers and apply it directly to the creation of tactile observation materials using 3D printing technology.

\section{Developed Software and Evaluation of Dot Size on a Sample Embossed Dot Graphic}

Upon considering these benefits and circumstances surrounding 3D-printed embossed dots graphics, the author designed this study to specifically take up the following challenge: develop a system that would convert data created using paper embossed dots graphics drawing software for a braille embosser into data that could be used with 3D CAD software. The conversion would allow for completely customization of the size and shape of every dot, freeing us from the limitations in dot type associated with the braille embosser. The author then sent the $3 \mathrm{D}$ CAD data that the system generated during the conversion to a 
consumer 3D printer and studied its replicability for molded objects and its effectiveness for use with tactile observation educational materials.

Software: For this study, the author developed software that would take data generated by the embossed dots graphics drawing program Edel and convert it into 3D CAD data. The called the conversion software Edl2scad. Edel is a piece of drawing software for designing embossed dots graphics for the ESA 721 [6] embosser. It can punch out three dot sizes. ESA 721 is able to emboss dots without typical cracks which are often caused by a mechanical embosser. By converting data so that it can be edited on OpenSCAD, users can take the drawings they've made on Edel and revise them in SCAD data format on OpenSCAD as solid objects. The small, medium, and large dots included in the Edel data are converted into solid hemispheres on the surface of circular truncated cones in accordance with their respective sizes. Figure 1 (leftside) shows an enlarged photo of the dots from an embossed dots graphics design that were produced using an ESA 721 embosser.

About the Evaluation Sample: The author used Edel to create a map, which he then studied to identify the qualities and characteristics of tactile graphics produced with the 3D embossed dots graphics process. More specifically, the author found a map showing the power relationships on the Italian peninsula during the 15th century, and used small, medium, and large dots to recreate it as an embossed dots graphic. Figure 1 (rightside) is given as an example of how the map he made was displayed on Edel.
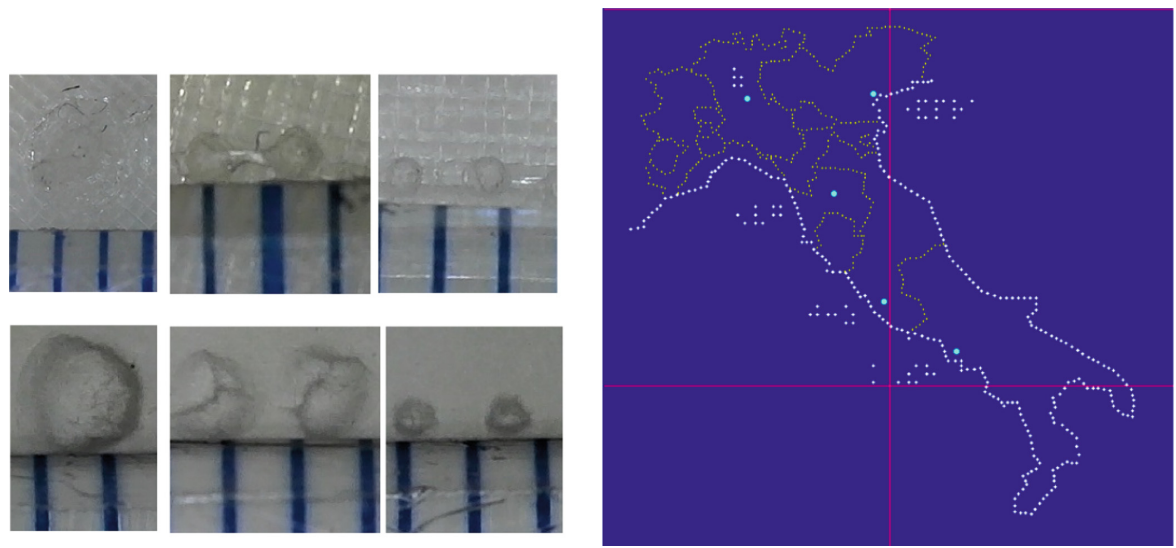

Fig. 1. Three dots embossed by ESA721 (up-left), generated by 3D printer (down-left) and the evaluated embossed dots map (right) 


\section{Physical Measurements and Tactile Observation Assessments}

Measuring and Evaluating Dot Size: The author used the same numbers to create a model in order to get a $3 \mathrm{D}$ embossed dots graphic that conformed to ESA 721 output standards. The measured values were then used to form dot modeling solids, and the author attempted to replicate the shape. The model that he actually produced ended up being smaller than the designated values. In response to these results, the author intentionally enlarged the sizes beyond those of the corresponding dots in the embossed model. He repeated this process until he ended up with dots of the same size on the 3D embossed dots graphic.

The author then made further adjustments to the size of the large dots until he was able to use a $3 \mathrm{D}$ printer to produce the embossed dots graphic originally intended for the ESA 721. The mechanical components in the ESA 721 are designed to produce a large dot size with a radius of one millimeter. Due to the performance of the hammer solenoid and characteristics of the printing paper, however, the radius of the large dots was actually limited to about $0.75 \mathrm{~mm}$. Because the $3 \mathrm{D}$ printer is not limited by these mechanical restrictions, it was able to create the originally intended one-millimeter large dots in the $3 \mathrm{D}$ embossed dot graphic. Table 1 shows the settings used in order to get an output that corresponded to the ESA 721 object.

Table 1. The settings and results of test printings

\begin{tabular}{|c|c|c|c|c|c|}
\hline \multirow[t]{4}{*}{ Dot type } & \multicolumn{5}{|c|}{ Radius (mm) } \\
\hline & \multirow{3}{*}{$\begin{array}{l}\text { ESA } 721 \\
\text { (reference) }\end{array}$} & \multicolumn{4}{|c|}{ Generated 3D embossed dots } \\
\hline & & \multicolumn{2}{|c|}{$\begin{array}{l}\text { Using the measured } \\
\text { values as settings }\end{array}$} & \multicolumn{2}{|c|}{$\begin{array}{l}\text { Attempting to make dots } \\
\text { originally intended for ESA }\end{array}$} \\
\hline & & Setting & Result & Setting & Result \\
\hline Large & 0.75 & 0.75 & 0.5 & 1.7 & 1 \\
\hline Medium & 0.5 & 0.5 & 0.45 & 0.55 & 0.5 \\
\hline Small & 0.25 & 0.25 & NA (not observed) & 0.3 & 0.35 \\
\hline
\end{tabular}

Tactile Observation Evaluations: Evaluations made via tactile observation were intensively conducted by the author, a visually impaired person with total blindness. Follow-up assessments were then conducted by other visually impaired people as a group discussion to corroborate these conclusions. The following lists feedback collected in the course of tactile observations. (1) The 3D models were not inferior to the embossed dots graphics produced on paper in terms of their expressive features (2) Changing the three dot sizes made it easier to distinguish them in the 3D model compared to the paper embossed dots graphics. The large dots in particular were greatly improved in terms of palpability. (3) The surface 
smoothness of the 3D model was inferior to that of the embossed dots graphic produced on paper. (4) The abbreviated city names written on the map in braille were not easy to read.

\section{Conclusions}

This study created and evaluated a system for using a 3D printer to create solid models corresponding to the embossed dots graphics produced on a braille embosser. The system was able to take embossed dots graphics data generated using embossed dots graphics drawing software and use it as-is to mold a threedimensional object. The author did discover a problem, however, that he did not give much weight to in the beginning: the dots did not sufficiently protrude when he used the exact size value for the paper embossed dots graphics to create the data for the 3D model.

Acknowledgments. This work was supported by KAKENHI (17H02005).

\section{References}

1. Teshima, Y., Matsuoka, A., Fujiyoshi, M., Ikegami, Y., Kaneko, T., Oouchi, S., Watanabe, Y., Yamazawa, K.: Enlarged skeleton models of plankton for tactile teaching. In: Miesenberger, K., Klaus, J., Zagler, W., Karshmer, A. (eds.) ICCHP 2010. LNCS, vol. 6180, pp. 523-526. Springer, Heidelberg (2010). https://doi.org/ 10.1007/978-3-642-14100-3_78

2. Götzelmann, T.: CapMaps. In: Miesenberger, K., Bühler, C., Penaz, P. (eds.) ICCHP 2016. LNCS, vol. 9759, pp. 146-152. Springer, Cham (2016). https://doi.org/10. 1007/978-3-319-41267-2_20

3. Serrano-Mira, J., et al.: Use of additive manufacturing to obtain moulds to thermoform tactile graphics for people with visual impairment. Procedia Manuf. 13, 810-817 (2017)

4. Braille Authority of North America, Canadian Braille Authority: Guidelines and Standards for Tactile Graphics (2011)

5. Edel and its related software. http://www7a.biglobe.ne.jp/ EDEL-plus/EdelDown Load.html. (in Japanese, English version of these software are also hosted)

6. ESA721 Ver'95. http://www.jtr-tenji.co.jp/products/ESA721_Ver95/. (in Japanese) 
Open Access This chapter is licensed under the terms of the Creative Commons Attribution 4.0 International License (http://creativecommons.org/licenses/by/4.0/), which permits use, sharing, adaptation, distribution and reproduction in any medium or format, as long as you give appropriate credit to the original author(s) and the source, provide a link to the Creative Commons license and indicate if changes were made.

The images or other third party material in this chapter are included in the chapter's Creative Commons license, unless indicated otherwise in a credit line to the material. If material is not included in the chapter's Creative Commons license and your intended use is not permitted by statutory regulation or exceeds the permitted use, you will need to obtain permission directly from the copyright holder.

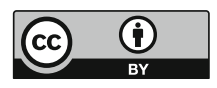

